\title{
Burn and plug: is it too much for the left atrial appendage?
}

\author{
Nicola Tarantino ${ }^{1} \cdot$ Jorge Romero $^{1} \cdot$ Xiao-Dong Zhang $^{1} \cdot$ David Briceño $^{1} \cdot$ Luigi Di Biase $^{1}$ (D)
}

Received: 9 September 2019 / Accepted: 9 October 2019 /Published online: 16 November 2019

(C) Springer Science+Business Media, LLC, part of Springer Nature 2019

Since our initial observations [1], there has been a growing interest in the left atrial appendage (LAA) as a target for the treatment of atrial fibrillation (AF). The BELIEF randomized controlled trial showed that in patients with long-standing persistent atrial fibrillation (LSPAF), 56\% of subjects were arrhythmia-free at 1-year follow-up after empirical LAA electrical isolation (LAAEI) compared with $28 \%$ of patients in the group $(P=0.001)$ [2]. Subsequently, also cryoballoon $(\mathrm{CB})$ ablation proved the safety and benefit of LAAEI $[3,4]$.

However, along with the enthusiasm of the new ablation strategy, serious concerns arose regarding the increased risk of thromboembolism (TE) originating from the non-contracting LAA. On the other hand the BELIEF trial [2] and two comprehensive meta-analyses $[5,6]$ reported reassuring results in terms of embolic stroke.

In our meta-analysis $(n=930)$, we showed no difference of TE in patients who underwent LAAEI compared with the control group $(0.2 \%$ vs. $2.1 \%, P=0.13)$ [6]. Furthermore, Yorgun et al. published a propensity score matched study assessing balloon-based LAAEI [4]. This study reported only one TE in the LAAEI group at 1-year follow-up in a patient who had discontinued oral anticoagulation (OAC). TEE (transesophageal echocardiogram) at 3- and 12-month follow-up showed no LAA thrombi. Conversely, two non-randomized studies by Rillig et al. in 2016 [7] and more recently by Kim et al. in 2018 [8] reported significantly higher risks of stroke and transient ischemic attacks (TIA) in patients post-LAAEI. In the study by Kim et al., there was an extremely high rate of TE $(23 \%)$. The authors themselves acknowledged that at least 5 out of $9(56 \%)$ patients with TE were off OAC and two were on warfarin with unknown INRs $(22 \%)$. These

Luigi Di Biase

dibbia@gmail.com

1 Albert Einstein College of Medicine, Montefiore Hospital, New York, NY, USA studies demonstrated that non-compliance with OAC is an important factor to experience TE events after LAAEI. Therefore, proper counseling about the necessity of strict compliance to OAC is required before and after LAAEI. Given the opposite and contradicting data, our group has recently reported the incidence of TE post-LAAEI cases "on" and "off" oral OAC [9]. Of the 1518 patients with abnormal LAA contractility at 6 months after LAAEI, 1086 remained on OAC, and the incidence of stroke in this population was 18 of $1086(1.7 \%)$, whereas the number of TE events in the off-OAC patients $(n=432)$ was 72 $(16.7 \%) ; p<0.001$. Of the 90 patients with stroke, 84 received left atrial appendage occlusion (LAAO) devices. At median 12.4 months (interquartile range 9.8 to 15.3 months) of device implantation, 2 (2.4\%) patients were on OAC because of high stroke risk or personal preference, whereas 81 patients discontinued OAC after LAAO device implantation without any TE events.

Although LAA closure devices are currently not recommended immediately after LAAEI due to considerable tissue inflammatory edema and consequently the potential risk of under-sizing the device, some groups have shown this might be actually feasible and presumptively the best option after LAAEI given the patients' poor compliance with OAC. Hence, a fundamental dilemma arises: if LAAEI preludes to occlusion, when is the optimal timing?

In a study reported in this issue of JICE, Kita et al. [10] investigated the safety and feasibility of concomitant circumferential LAAEI with radiofrequency (RF) and occlusion (LAAO) with WATCHMAN device, to prevent TE from the mechanically inert appendage, and also to reduce the risks related to a subsequent standalone procedure. The authors observed not only that LAAEI increases the AF-free survival at 1 year in $94.5 \%$ of their sample but also that simultaneous LAA closure was successful in $100 \%$ of the cases with no major periprocedural complications. After LAAEI/LAAO, neither stroke nor TIA occurred in the whole cohort of 42 patients while they were off $\mathrm{OAC}$, despite the mean $\mathrm{CHA}_{2} \mathrm{DS}_{2}$-Vasc score corresponding to an estimated $3.2 \%$ annual risks of TE events. 
These findings are consistent with previous elegant observations. A pioneering experiment on a canine model by Panniker et al. demonstrated that combined ostial LAAEI and LAAO was doable, with durable LAA disconnection and no complications related to periprocedural implant [11]. The ex vivo analysis showed that WATCHMAN was symmetrically expanded and fully endothelialized at 45 days, with no signs of dislodgment in 8 animals. Concomitant LAAEI and occlusion as part of conventional AF ablation was also initially tested in humans by the same group, who evaluated this concept in patients with LSPAF undergoing RF ablation [12]. LAAO device (Watchman, Boston Scientific, MA, USA) was successfully implanted in all patients with no major periprocedural complications. Imaging at 45 days and 9 months confirmed satisfactory device position and excluded pericardial effusion. Warfarin was suspended in 19 of the 20 (95\%) patients at 3 months. Notably, in both animal and human studies, circumferential ablation of the LAA did not alter the size of the ostium; but the LAA orifice diameter significantly increased $[11,12]$. The hypothesis illustrated by the authors is interesting (the volume of fluid infused during the procedure probably restored the real compliance of the LAA in sinus rhythm), and the immediate technical implications are much more meaningful. Indeed, one of the major reluctance to perform simultaneous LAAEI/LAAO is represented by the fact that post-ablation tissue edema could reduce the real size of the ostium with consequent ostium-device mismatch, and eventually increase the risk of device dislodgment, embolization and peri-device leaks. Nevertheless, both Panniker and Kita revealed that the incidence of severe leaks was minimal and transient (5\% and $2 \%$ respectively), whereas only one patient overall experienced a clear device migration, but none in both cohorts reported device embolization or TE at followup $[10,12]$.

Another group reported on concomitant cryoballoon ablation and percutaneous closure of LAA in patients with AF [13]. LAA occlusion was carried out by using two occluder devices (Amplatz Cardiac Plug, ACP, St. Jude Medical, MN, USA, and Watchman, Boston Scientific, MA, USA). Eightysix percent of patients had complete sealing; $14 \%$ of patients showed a residual flow $(<5 \mathrm{~mm})$ at first TEE check, while at 1 -year TEE residual flow was detected in 3 patients. No device-related complications or clinical thromboembolic events occurred.

Thus, is the combined "ablate and occlude" treatment the best strategy able to mitigate the short-midterm risk of stroke after LAAEI, and also to diminish risks and costs related to a second elective procedure? There are some important caveats worth discussing.

First, the aforementioned findings are not extensively applicable. The reported data, albeit promising, are based on single-center experiences with a small number of patients with no control groups. Results are limited to the use of RF and CB to isolate the LAA associated with the deployment exclusively of WATCHMAN and Amplatz Cardiac Plug, ACP. The LAA can be successfully isolated with $\mathrm{CB}[3,4]$; nevertheless, the extent of edema caused by hypothermal injury is remarkable [14] and the risk to compromise the simultaneous LAAO device should not be ignored in this setting, despite the results of Fassini el al [13]. Second, according to Kita et al. [10], there were some objective impediments to TEE measurements of the LAA ostium. In fact, the tissue edema shadowed the LAA edges, impairing the spatial resolution of the ostial diameter, which has major implications when sizing the device. The authors obviate this issue by systematically upsizing the implanted WATCHMAN, and-as they reported - this strategy was successful. Probably, this inconvenience may represent a major limitation, especially for borderline ostial sizes for which an appropriate choice of the occluder can be determinant. We agree with Kita et al. that this problem can also be overcome with the support of alternative imaging like LAA angiography (as in their work), but we consider that intracardiac echo (ICE) or pre-procedural cardiac CT offer multiplanar views and can be therefore more valid modalities. On the other side, since the LAA volume is dependent upon the current fluid status, pre-ablation TEE may not offer reproducible measures in this context. It is noteworthy to consider another merely technical aspect: how much safe is to recapture the WATCHMAN, given the degree of post-ablation inflammation? To this question, we actually do not have answers; however, we deem that combining these procedures should restrict the acquired confidence in maneuverability of the WATCHMAN delivery system.

A third point pertains to acute and late LAA electrical reconnection. As stated elsewhere [15], assessment of persistent LAAEI is recommended before LAA occlusion. About $85 \%$ of LAA reconnects within $60 \mathrm{~min}$ [12]; thus, we suggest to test dormant conduction with adenosine or isoproterenol, and to wait a reasonable time between isolation and LAAO (i.e., 30 min). This, nonetheless, implies longer procedural time, which should be evaluated in advance taking into account also the anesthesia-related risk. Late LAA reconnections, instead, were observed in $37 \%$ of the redo cases in the BELIEF trial [2]. Likewise, Reissmann et al. reported a $27 \%$ reconnection rate after a median of 3 months in redo cases [16]. Panikker et al. used the absence of far-field LAA in the LSPV (left superior pulmonary vein) as surrogate marker of persistent LAAEI [12], and in the present study, LAA electrograms were clearly recorded over the device in one redo case 3 months after the index procedure, with successful atrial tachycardia ablation in proximity of the anterior border of the LAA. On the other hand, in the vacuum of information regarding the safety and feasibility of mapping and ablation around the LAA ostium with an LAAO device in situ, we theoretically think that such an approach might damage the integrity of the device. Therefore, from this standpoint, it would be perhaps 
preferable to perform LAAO implantation as deferred procedure once patient has remained in sinus rhythm off antiarrhythmic drugs for at least 3-6 months.

Furthermore, device-related thrombosis (DRT) was noted in approximately $7 \%$ of the patients who underwent TEE at 3-6 months [10]. The current protocol for WATCHMAN device is to perform 1-year follow-up TEE to reassess for DRT. Interestingly, a PROTECT AF sub-study disclosed that $32 \%$ of the patients had at least some degree of peri-device leak at 12 months suggesting that this phenomenon was very common [17]. However, the incidence of TE events associated with DRT has repetitively been reported as very low [18]. Likewise, consistent DRT resolution has been reported after treatment with OAC. However, in the PROTECT AF and the CAP registry, $15 \%$ of DRTs were associated with clinical strokes [19]. More recently, Fauchier et al. corroborated that DRT incidence is as high as $7.7 \%$ [20], which is similar to the current study [10]. More importantly, the same authors showed that DRTs are frequently associated with embolice stroke (15\%). The association between DRT and lack of appropriate post-procedural DAPT or OAC and consequently the TE appear robust.

Lastly, a cost-benefit analysis of the one-stage hybrid technique should be considered. Since there are no data about it, we can speculate that on account of the multiple procedure rule, combining two expensive procedures can be disadvantageous in terms of reimbursement. Yet, one could argue that simultaneous LAAEI/LAAO may reduce the cost of two distinct procedures, as well as the overall length of in-hospital stay, the total duration of OAC and antiarrhythmic medications, with consequent savings in terms of pharmacological treatment and of any possible re-hospitalization secondary to OAC adverse events.

In conclusion, we congratulate Kita et al. for their outstanding contribution. They may have paved the way to a new systematic approach, which, although with intrinsic limitations, can become the standard of care for the selected strata of patients. Identifying high risk subjects (i.e., elevated $\mathrm{CHA}_{2} \mathrm{DS}_{2}$-Vasc score, labile OAC adherence or with significant bleeding profile) might represent the key for the success of the simultaneous technique. Nonetheless, in the absence of prospective randomized controlled trials comparing LAAO device vs. OAC following LAAEI, the incremental benefit of the combined procedure is still unknown and, given the substantial rate of late LAA reconnections, LAAO device implantation should in our opinion be performed once long-term sinus rhythm has been achieved.

\section{Compliance with ethical standards}

Conflict of interest Dr. Di Biase is a consultant for Biosense Webster, Stereotaxis, Boston Scientific, and Abbott Medical. He received speaker honoraria/travel from Medtronic, Pfizer, Bristol Myers Squibb, and Biotronik. The remaining authors have no disclosures.

\section{References}

1. Di Biase L, Burkhardt JD, Mohanty P, Sanchez J, Mohanty S, Horton R, et al. Left atrial appendage: an underrecognized trigger site of atrial fibrillation. Circulation. 2010;122(2):109-18. https:// doi.org/10.1161/CIRCULATIONAHA.109.928903.

2. Di Biase L, Burkhardt JD, Mohanty P, Mohanty S, Sanchez JE, Trivedi $\mathrm{C}$, et al. Left atrial appendage isolation in patients with longstanding persistent AF undergoing catheter ablation: BELIEF Trial. J Am Coll Cardiol. 2016;68(18):1929-40. https://doi.org/10. 1016/j.jacc.2016.07.770.

3. Bordignon S, Chen S, Perrotta L, Bologna F, Nagase T, Konstantinou A, et al. Durability of cryoballoon left atrial appendage isolation: acute and invasive remapping electrophysiological findings. Pacing Clin Electrophysiol. 2019;42(6):646-54. https:// doi.org/10.1111/pace.13690.

4. Yorgun H, Canpolat U, Kocyigit D, Coteli C, Evranos B, Aytemir $\mathrm{K}$. Left atrial appendage isolation in addition to pulmonary vein isolation in persistent atrial fibrillation: one-year clinical outcome after cryoballoon-based ablation. Europace. 2017;19:758-68. https://doi.org/10.1093/europace/euz232.

5. Friedman DJ, Black-Maier EW, Barnett AS, Pokorney SD, AlKhatib SM, Jackson KP, et al. Left atrial appendage electrical isolation for treatment of recurrent atrial fibrillation: a meta-analysis. JACC Clin Electrophysiol. 2018;4(1):112-20. https://doi.org/10. 1016/j.jacep.2017.07.007.

6. Romero J, Michaud GF, Avendano R, Briceño DF, Kumar S, Carlos Diaz J, et al. Benefit of left atrial appendage electrical isolation for persistent and long-standing persistent atrial fibrillation: a systematic review and meta-analysis. Europace. 2018;20(8):1268-78. https://doi.org/10.1093/europace/eux372.

7. Rillig A, Tilz RR, Lin T, Fink T, Heeger CH, Arya A, et al. Unexpectedly high incidence of stroke and left atrial appendage thrombus formation after electrical isolation of the left atrial appendage for the treatment of atrial tachyarrhythmias. Circ Arrhythm Electrophysiol. 2016;9(5):e003461. https://doi.org/10. 1161/CIRCEP.115.003461.

8. Kim YG, Shim J, Oh SK, Lee KN, Choi JI, Kim YH. Electrical isolation of the left atrial appendage increases the risk of ischemic stroke and transient ischemic attack regardless of post isolation flow velocity. Heart Rhythm. 2018;15(12):1746-53. https://doi.org/10. 1016/j.hrthm.2018.09.012.

9. Di Biase L, Mohanty S, Trivedi C, Romero J, Natale V, Briceno D, et al. Stroke risk in patients with atrial fibrillation undergoing electrical isolation of the left atrial appendage. J Am Coll Cardiol. 2019;74(8):1019-28. https://doi.org/10.1016/j.jacc.2019.06.045.

10. Kita K, Carlson S, Huntsinger M, Tun H, Sohn J, Doshi RN. Safety and feasibility of combined atrial fibrillation ablation and left atrial appendage occlusion after left atrial appendage electrical isolation. J Interv Card Electrophysiol. 2019. https://doi.org/10.1007/s10840019-00603-1.

11. Panikker S, Virmani R, Sakakura K, Kolodgie F, Francis DP, Markides V, et al. Left atrial appendage electrical isolation and concomitant device occlusion: a safety and feasibility study with histologic characterization. Heart Rhythm. 2015;12(1):202-10. https://doi.org/10.1016/j.hrthm.2014.09.010.

12. Panikker S, Jarman JW, Virmani R, Kutys R, Haldar S, Lim E, et al. Left atrial appendage electrical isolation and concomitant device occlusion to treat persistent atrial fibrillation: a first-in-human safety, feasibility, and efficacy study. Circ Arrhythm Electrophysiol. 2016;9(7). https://doi.org/10.1161/CIRCEP.115.003710.

13. Fassini G, Conti S, Moltrasio M, Maltagliati A, Tundo F, Riva S, et al. Concomitant cryoballoon ablation and percutaneous closure of left atrial appendage in patients with atrial fibrillation. Europace. 2016;18:1705-10. https://doi.org/10.1093/europace/euw007. 
14. Bordignon S, Perrotta L, Fürnkranz A, Bologna F, Schmidt B, Chun KR. Durable single shot cryoballoon isolation of the left atrial appendage followed by percutaneous left atrial appendage closure. Circ Arrhythm Electrophysiol. 2015;8(3):751-2. https://doi.org/ 10.1161/CIRCEP.115.002885.

15. Romero J, Di Biase L. Left atrial appendage electrical isolation for persistent atrial fibrillation: radiofrequency vs cryoballoon ablation. Pacing Clin Electrophysiol. 2019;42(6):655-7. https://doi.org/10. 1111/pace.13689.

16. Reissmann B, Rillig A, Wissner E, Tilz R, Schlüter M, Sohns C, et al. Durability of wide-area left atrial appendage isolation: results from extensive catheter ablation for treatment of persistent atrial fibrillation. Heart Rhythm. 2017;14(3):314-9. https://doi.org/10. 1016/j.hrthm.2016.11.009.

17. Viles-Gonzalez JF, Kar S, Douglas P, Dukkipati S, Feldman T, Horton R, et al. The clinical impact of incomplete left atrial appendage closure with the Watchman Device in patients with atrial fibrillation: a PROTECT AF (percutaneous closure of the left atrial appendage versus warfarin therapy for prevention of stroke in patients with atrial fibrillation) substudy. J Am Coll Cardiol. 2012;59(10): 923-9. https://doi.org/10.1016/j.jacc.2011.11.028.
18. Main ML, Fan D, Reddy VY, Holmes DR, Gordon NT, Coggins TR, et al. Assessment of device-related thrombus and associated clinical outcomes with the WATCHMAN left atrial appendage closure device for embolic protection in patients with atrial fibrillation (from the PROTECT-AF Trial). Am J Cardiol. 2016;117:1127-34. https://doi.org/10.1016/j.amjcard.2016.01.039.

19. Reddy VY, Holmes D, Doshi SK, Neuzil P, Kar S. Safety of percutaneous left atrial appendage closure: results from the Watchman left atrial appendage system for embolic protection in patients with AF (PROTECT AF) clinical trial and the continued access registry. Circulation. 2011;123:417-24. https://doi.org/10.1161/ CIRCULATIONAHA.110.976449.

20. Fauchier L, Cinaud A, Brigadeau F, Lepillier A, Pierre B, Abbey S, et al. Device-related thrombosis after percutaneous left atrial appendage occlusion for atrial fibrillation. J Am Coll Cardiol. 2018;71:1528-36. https://doi.org/10.1016/j.jacc.2018.01.076.

Publisher's note Springer Nature remains neutral with regard to jurisdictional claims in published maps and institutional affiliations. 